\title{
REFERENCE POINTS IN MAGNETIC MEASUREMENTS
}

\author{
Ömer Can DEMIRTAS \\ University of Dicle, Medical Faculty, Department of Biophysics \\ 21280 Diyarbakır - Türkiye. email : $\operatorname{demirtas} a$ dicle.edu.tr \\ Rauf MIRZABABAYEV \\ University of Gaziantep, Electrical and Electronics Engineering Department \\ 27310 Gaziantep - Türkiye. email : mirza $a$ gantep.edu.tr
}

\begin{abstract}
It is widely assumed that the magnetic phase transition temperatures have constant values. Application of the formalism of the high accuracy spectroscopic method shows that the Curie points used for the calibration processes in magnetic measurements are not uniquely defined, but are related to the time scale of the measuring method.
\end{abstract}

\section{INTRODUCTION}

Before any measurement is provided measuring instruments should be calibrated. In temperature measurements certain double or tripled points, that is the phase transition temperatures are used. For example, the melting point of ice and the boiling point of water are widely used as a reference points to set the various temperature scales

In measuring the magnetic properties of materials, the magnetic phase transition temperatures known as the Curie points are used. It is generally assumed that the phase transitions are inherently connected with the physical properties of materials and do not depend on the applied methods of measurements. The differences in the Curie points which are observed when measured by various methods usually are referred to as the trivial statistic and systematic errors of measurements. Consequently, they didn't attract much attention and left without any explanation even when the differences are too big $[1,2]$. Application of the high accuracy spectroscopic methods to the measurement processes makes it possible to throw a new look at all these phenomena. The aim of this work is to apply some new approaches to the measurement process using the scaling theory and the formalism of Mossbauer spectroscopy.

\section{CRITICAL PHENOMENA}

Ferromagnetic systems are classic examples of systems exhibiting cooperative phenomena and as such are commonly used to demonstrate phase transitions and critical phenomena. Simple ferromagnets can be imagined as consisting of spins located at the lattice sites of a system with the neighbouring spins tending to align. At high temperatures with no applied magnetic field, the thermal fluctuations overcome the tendency for the spins to align and the system has no magnetic ordering. However, below the Curie temperature long range alignment occurs between the spins throughout the system. 


\subsection{The ferromagnet as the critical system}

The thermodynamic properties of ferromagnets, such as the magnetisation and the specific heat, are well behaved for temperatures away from the Curie temperature and for all temperatures when an external magnetic field is applied. However, their behavior is singular with a value approaching infinity at the critical point. Critical points thus can be characterised mathematically as non-analytic points in otherwise analytic systems. According to the scaling theory, the behavior of a system near its critical point should depend on only a small number of general characteristics. If the proper analogous quantities and parameters can be found in the various systems with critical points, the same formalism can be applied to each, and the results in one will contribute to the understanding of the others

A simple ferromagnet consists of a collection of equivalent magnetic moments, fixed in spatial position but not necessarily forming a regular lattice. Each spin is free to orient itself in any direction but there is an exchange interaction which tends to line up neighbouring spins. The important external influences on such a system are the temperature $\mathrm{T}$ and the external magnetic field $\mathrm{H}$

\subsection{The scaling theory}

In an ideal ferromagnet with no external field, the spins are oriented in the same direction at $\mathrm{T}=0 \mathrm{~K}$. The degree of ordering in the system can be characterised by the order parameter $<\sigma$ where $\sigma$ is the reduced magnetic moment $(|\sigma| \leq 1)$. When the ferromagnet is heated up the thermal energy $\mathrm{kT}$ becomes sufficient to excite spin waves and spin flips leading to a decrease in $\langle\sigma\rangle$. If the temperature is not too high, the spin waves have negligible interactions and can be treated as free excitations. This is seen in the $T^{3 / 2}$ dependence for the magnetisation of such systems. The interaction of the excitations increases as the temperature is increased, resulting in a faster decrease in the ordering of the magnet. As the critical temperature is approached the interaction between spin waves becomes strong enough that the excitations are correlated over an increasing range characterised by the correlation length $\xi$. It depends on the temperature as [3]

$$
\xi(T)=\xi_{0}\left|\left(T-T_{c}\right) / T_{c}\right|^{-v}
$$

here $\xi$ - is the amplitude of the correlation length, $v$ - is the critical exponent

The correlation length for the excitations becomes infinite and the ordering parameter

$\sigma>$ becomes zero at the critical point temperature. Increasing the temperature further leads to a thermal breaking up of the correlation and a decrease in the correlation length $\xi$. However $\sigma \sigma$ remains zero; there is no macroscopic order above the critical temperature.

Within the correlation length all atoms fluctuate coherently. Their behavior is the same as in the magnetic microcrystals of a single domain.

\section{MAGNETIC ANISOTROPY}

In the absence of an external magnetic field, the magnetization direction of a large nagnetically ordered crystal is along an easy direction. The easy directions are defined as the low energy directions of the spin system and are separated by the energy barriers. When the size of magnets decreases drastically, the thermal energy may become comparable to the anisotropy energy. As a result, in small particles of a magnetically ordered material, the relative orientation of atomic spins is specified, but the entire spin system rotate in space without changing in energy

The magnetic uniaxial anisotropy is given by the expression [4]

$$
\mathbf{E}(\theta)=\mathbf{K V} \sin ^{2} \theta
$$


where $\mathrm{K}$ - is the anisotropy energy constant, $\mathrm{V}$ - is the volume of the particle, and $\theta$ - is the angle between magnetization direction and the easy direction of magnetization. According to the above equation, two energy minima at $\theta=0$ and $\theta=\pi$ are separated by an energy barrier equal to $\mathrm{KV}$.

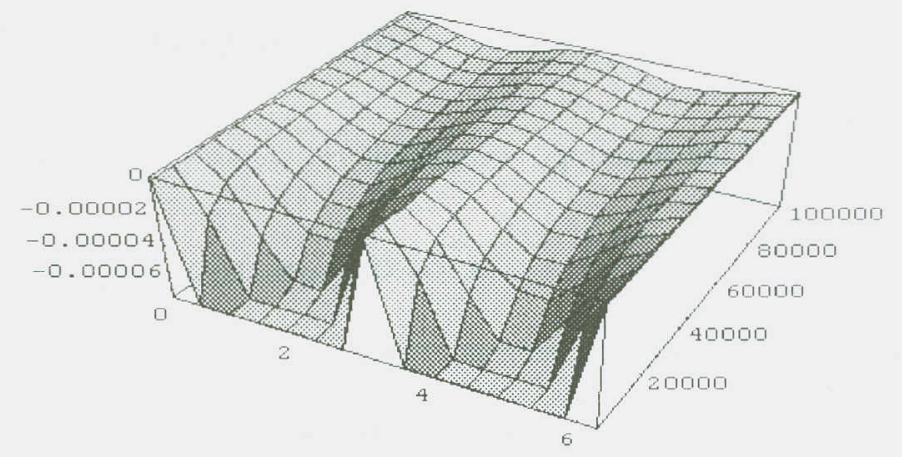

Figure 1. 3-Dimensional diagram for the normalized energy of magnetic quasiparticles

In the presence of an external magnetic field $\mathrm{H}$ along the symmetry axis, the energy of a ferromagnetic particle is given by

$$
\mathbf{E}(\theta)=K V \sin ^{2} \theta-H M_{S} V \cos \theta
$$

For $\mathrm{H}<2 \mathrm{~K} / \mathrm{M}_{\mathrm{S}}$ there are two minima at $\theta=0$ and $\theta=\pi$, but for larger applied fields the minimum at $\theta=\pi$ disappears. Figure 1 . shows the normalized magnetization as a function of temperature and volume of a quasiparticle. The figure has been drawn by means of the standard software Mathematica 2.2.

The probability that the magnetization vector of a small particle forms an angle between $\theta$ and $\theta+d \theta$ with an easy direction is given by

$$
f(\theta) d \theta=\frac{\exp [-E(\theta) / k T] \sin \theta d \theta}{\left.\int_{0}^{\pi} \exp \mid-E(\theta) / k T\right] \sin \theta d \theta}
$$

Here $\mathrm{k}$ is Boltzmann's constant and T is the temperature. When KV are very large compared to $k T, f(\theta) \approx 0$ except at the energy minima. The magnetization can then be considered fixed in one of these directions. At smaller values of $\mathrm{KV} / \mathrm{kT} \mathrm{f}(\theta)$ broadens around its minima. For $\mathrm{KV} / \mathrm{kT}$ $\leq 1$ the magnetization has a significant probability of surmounting the energy barrier separating the minima under the relaxation process.

The superparamagnetic relaxation time varies with temperature as

$$
\tau=\tau_{0} \exp (\mathbf{K V} / \mathbf{k T})
$$

$\tau$ is the order of $10^{-10} \mathrm{~s}$. 
The magnetization of a ferromagnetic microcrystal, averaged over a time that is long compared to the characteristic time of the fluctuations, can be derived by using equation (4) and is given by

$$
<\mathbf{M}>=\mathbf{M}_{\mathbf{s}} \frac{\int_{0}^{\pi} \exp [-\mathbf{E}(\theta) / \mathbf{k T}] \sin \theta \mathrm{d} \theta}{\int_{0}^{\pi} \exp [-\mathbf{E}(\theta) / \mathbf{k T}] \sin \theta \mathrm{d} \theta}
$$

When the effect of the anisotropy is negligible in contrast to the influence of the applied field, equation (6) reduces to

$$
<\mathbf{M}>=\mathbf{M}_{\mathrm{S}} \mathbf{L}(\mu \mathbf{H} / \mathbf{k T}),
$$

where $\mu=M_{S} V$ is the magnetic moment of the particle and

$$
\mathbf{L}(\mu \mathbf{H} / \mathbf{k T})=\operatorname{coth}(\mu \mathbf{H} / \mathbf{k T})-(\mathbf{k T} / \mu \mathbf{H})
$$

is the classic Langevin Function. In the limit of low and the high fields $\mathrm{L}(\mu \mathrm{H} / \mathrm{kT})$ is given by

$$
\begin{aligned}
& \mathbf{L}(\mu \mathbf{H} / \mathbf{k T})=(\mu \mathbf{H} / 3 \mathbf{k T})(\mu \mathbf{H} / \mathbf{k T})<<1 \\
& \mathbf{L}(\mu \mathbf{H} / \mathbf{k T})=1-(\mathrm{kT} / \mu \mathrm{H}), \quad(\mu \mathbf{H} / \mathbf{k T})>1
\end{aligned}
$$

In studies of relaxation phenomena it is important to realise that the result of measurement depends on the time scale of the experimental technique compared to the time scale of the fluctuation phenomena. For example, if no applied field is present, then according to equation (6) the average magnetization is zero. However, if the superparamagnetic relaxation time is long compared to the observation time, a finite value of the magnetisation is measured. If the correlation time of the magnetic excitation is short compared to observation time, the measured magnetisation is given by

$$
M(V, T)=M_{s}(T)<\cos \theta>_{T}
$$

Where $<\cos \theta>_{\mathrm{T}}$ is the thermal average of $\cos \theta$ near one of the minimum. Setting $\mathrm{kT} / \mathrm{KV}=\beta$

$$
\int_{0}^{\pi / 2} \exp \left|(1 / \beta) \sin ^{2} \theta\right| \cos \theta \sin \theta d \theta
$$

$$
<\cos \theta>_{\mathrm{T}}=\frac{}{\int_{0}^{\pi / 2} \exp \left|(1 / \beta) \sin ^{2} \theta\right| \cos \theta \sin \theta d \theta}
$$

$$
=\frac{\beta^{1 / 2} \exp (1 / \beta)-1}{2 \int_{0}^{\beta-1 / 2} \exp \left(x^{2}\right) d x}
$$


In the low temperature limit $(\mathrm{KV} / \mathrm{k}, \mathrm{l})$ it is found that

$$
<\cos \theta>_{\mathrm{T}}=1-\mathrm{kT} / 2 \mathrm{KV}
$$

The diagrammed form of eq. $(11,12)$ is given in Fig. 2. It was plotted using the Microsoft Excel 97. Initially, the numerical values were calculated using the computer program written in QBASIC 7.0 programming language. Then the results of calculations were verified by the direct solution of integrals applying the standard software Mathematica 2.2

Figure 2 shows that if one takes into account the fluctuation phenomena in the critical region, then other conditions being equal, the measured value of the magnetization depends on the correlation length of fluctuations. In other words, the correlation length $\xi$ defines the relaxation time of atomic spins. Magnetization gradually drops to a steady state value approximated by the low temperature limit given in eq. (13). Thus, the Curie temperature is not uniquely defined, but is related to the time scale of the experimental technique used for the studies of magnetic properties. For the first time for bulk magnets this was observed in [5]

Each method of measurement is characterized by its own time scale defined by the physical principle of measurement. The time scale for observation of magnetically split Mossbauer spectra is approximately given by the Larmor precession time $\tau_{1}$ of the nuclear masnetic moment. For the susceptibility method which is widels ased for the magnetic measurements, the time scale is of order of second.

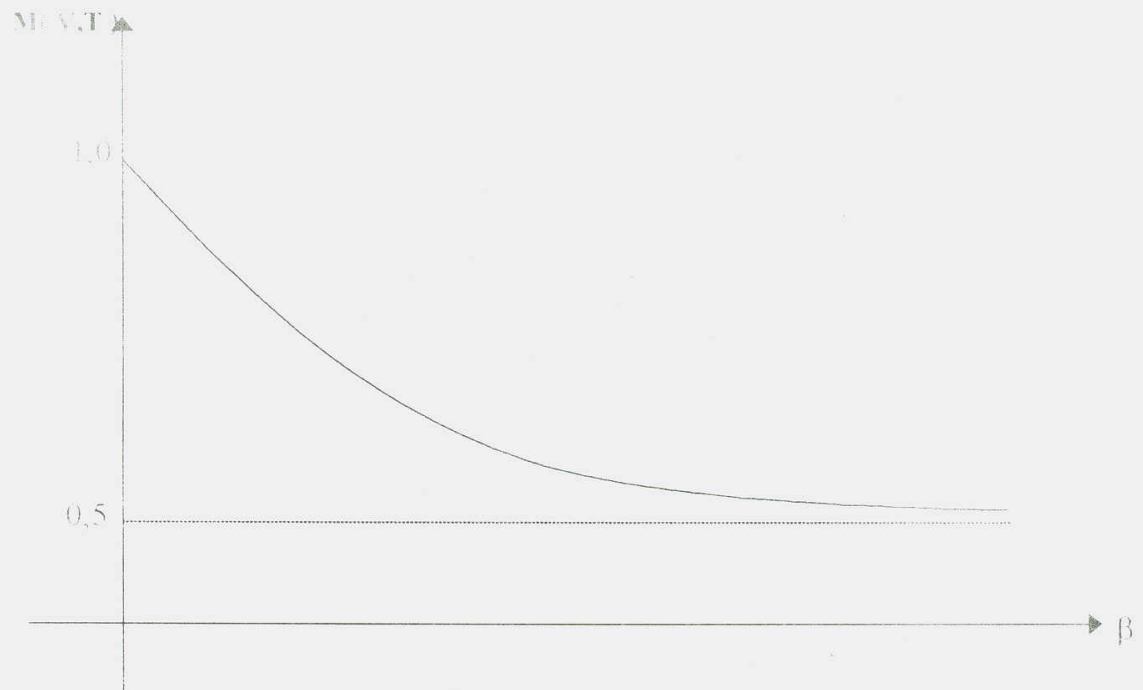

Figure 2. Normalized value for measured magnetization versus the time scale of the measuring method 


\section{CONCLUSION}

Magnetic phase transition temperatures depend on not only the intrinsic properties of materials but also the relation between the relaxation time and the characteristic times of the applied method of measurements. When magnetic material is measured by two different methods then the observed critical points, unlike the widely adopted view, can differ from each other.

\section{REFERENCES}

[1]. Long J.R., Mattozzi R.W., Journal of Applied Physics, 1984, v.55, N 6, p. 2359.

[2]. Kamzin A.S., Grigor'ev L.A., JETP Lett. ,vol. 57, N 9.

[3]. Fisher M.E., Journal of Vacuum Science Technology, 1973, N5, p.665

[4]. MØrup S., Dumesic J.A., Topsoe H., Application of Mossbauer Spectroscopy, 1980, N.Y., Acad Press., pp. 1-53

[5]. Mirzababayev R.M., McGrath R.D., Walker J.C., Journal de Physique, 1979, v.40, N 3. p.C.2-216 